\title{
HALLAZGO INCIDENTAL DE UN QUIMIODECTOMA EN UN CANINO BULLDOG INGLÉS
}

\author{
INCIDENTAL FINDING OF A CHEMODECTOMA IN A CANINE ENGLISH BULLDOG
}

\author{
Ávalos Ruíz Díaz A ${ }^{1}$, Maidana Moreno LG $^{1}$, Suárez $\mathrm{ME}^{1}$, González $\mathrm{A}^{1}$, Bellenzier $\mathrm{O}^{2}$, Sosa $\mathrm{G}^{2}$. \\ ${ }^{1}$ Departamento de Ciencias Patológicas, Facultad de Ciencias Veterinarias - Universidad Nacional de Asunción - San Lorenzo - \\ Paraguay \\ ${ }^{2}$ Estudiante de Grado, Facultad de Ciencias Veterinarias - Universidad Nacional de Asunción - San Lorenzo - Paraguay
}

\begin{abstract}
RESUMEN. En el presente estudio se describen hallazgos macroscópicos y microscópicos de un quimiodectoma en un canino, Bulldog inglés, hembra, de 6 años con muerte súbita y diagnóstico presuntivo de envenenamiento. El cadáver del animal se remitió al Departamento de Ciencias Patológicas de la Facultad de Ciencias Veterinarias (FCV) - Universidad Nacional de Asunción (UNA) para el estudio post-mortem. La necropsia reveló una protuberancia única, irregular $(4 \times 2,5 \mathrm{~cm})$ localizada en la base del corazón, periférica a la aorta, invadiendo adventicia e infiltrando miocardio auricular izquierdo con necrosis focal central. Al examen histopatológico de la protuberancia se constató una población celular compuesta por células poligonales, redondeadas y ovales con citoplasma granular, características histológicas de células quimiorreceptoras neoplásicas.
\end{abstract}

Palabras clave: quimiodectoma, cuerpo aórtico, canino, corazón.

\begin{abstract}
The present study describes macroscopic and microscopic chemodectoma findings in a canine English Bulldog , female, 6 years with sudden death and suspicious of poisoning. The animal was referred for post-mortem evaluation to the Pathology Sciences Department of Veterinary Faculty National University of Asunción (UNA) . The necropsy revealed an unique irregular protuberance $(4$ × 2.5 $\mathrm{cm}$ ) located at the heart base external to the aorta, invading adventicia and infiltrating left atrium myocardium with focal central necrosis. Histopathological examination showed polygonal, round and oval cells with granular cytoplasm, histologic characteristics of neoplastic chemoreceptor cells.
\end{abstract}

Keywords: chemodectoma, aortic body, canine, heart.

doi: http://dx.doi.org/10.18004/compend.cienc.vet. 2015.05.01.31-35

Dirección para correspondencia: Prof. Dr. Andrés Ávalos Ruíz Díaz - Facultad de Ciencias Veterinarias - Universidad Nacional de Asunción, Casilla de Correo Nº 1061 - Ruta Mcal. Estigarribia km 10,5 - Campus Universitario - San Lorenzo, Paraguay.

E-Mail: aavalos@vet.una.py

Recibido: 05 de marzo de 2015 / Aceptado: 17 de junio de 2015 


\section{INTRODUCCIÓN}

Los paragangliomas del cuerpo aórtico y carotídeo representan neoplasias raras que se originan de células neuroendócrinas del paraganglia del sistema parasimpático. Están compuestos por células no-cromafines originadas de la cresta neural. Este grupo de células está asociado al sistema nervioso autónomo, de donde también derivan el ganglio simpático y la médula adrenal (1). Ocurren principalmente en el cuerpo aórtico y carotídeo y menos frecuentemente del glomus pulmonar, glándula adrenal o sitios ectópicos. Los cuerpos aórtico y carotídeo son órganos quimiorreceptores sensitivos a variaciones del pH sanguíneo, tensión de oxígeno y contenido de dióxido de carbono, de modo que auxilian en la regularización de la respiración y la circulación $(2,3,4)$. Basados en esta función, estos tumores fueron llamados también quimiodectomas. Desde el punto de vista embriológico la nomenclatura más apropiada es paraganglioma (1). Aunque el origen embrionario de los órganos quimiorreceptores no sea precisamente conocido, existe considerable evidencia de que se originan de células mesodérmicas perivasculares que son invadidas por células de origen neuroectodérmico.

Los órganos quimiorreceptores en general, contienen dos tipos principales de células: las neuroendócrinas (quimiorreceptoras, glómicas o tipo I) y las de soporte o sustentaculares (capsulares o tipo II). Las células tipo I son redondeadas u ovoides, con núcleos pálidos y redondos, y poseen un citoplasma rico en vesículas de secreción (catecolamina y serotonina). Las células tipo II son consideradas de soporte y naturaleza neuroglial. Integradas al patrón histológico típico de este paraganglia existen un número variable de células del tejido conjuntivo, células vasculares, células de Schwann, fibras nerviosas mielínicas y amielínicas y neuronas intrínsecas (5).

Estos tumores comúnmente surgen del cuerpo aórtico, especialmente en caninos y representan el 7\% del total de casos de tumores cardíacos primarios (6). La frecuencia de este tipo de tumores se incrementa con la edad y generalmente la mayoría de los casos ocurre, en perros de seis años o más. Las razas que frecuentemente son diagnosticadas con este tipo de neoplasia, son las braquiocefálicas como los Bulldogs inglés y francés, Boston terrier y Bóxer (7).

La etiología aún se desconoce, no obstante la predisposición de los cánidos a padecer este tumor podría ser resultado de una hipoxia crónica debida al Síndrome de las vías aéreas de los braquiocefálicos (BAS); caracterizado por presencia de paladar blando alargado, narinas estenóticas y lesiones en tejido blando faríngeo a causa del escaso desarrollo rostral del cráneo. Estas alteraciones causarían una resistencia al flujo de aire en las vías aéreas superiores lo que ocasionaría el incremento de la presión negativa durante la inspiración resultando en fenómenos secundarios como el colapso laríngeo. Las lesiones del tejido blando derivarían en una hipoxia crónica que conduciría a una hiperplasia de los quimiorreceptores, pudiendo transformarse en una neoplasia de las mismas $(5,8)$.

Los signos clínicos debidos a la presencia de este tipo de neoplasia, incluyen insuficiencia cardíaca congestiva con hemorragia, tos, disnea, vómitos y cianosis. Los cambios físicos post-mortem observados incluyen hidrotórax, derrame pericárdico, ascitis, edema subcutáneo, congestión hepática y pulmonar $(9,10)$.

El objetivo del presente trabajo fue describir los hallazgos macroscópicos y microscópicos de un tumor aórtico en un canino de la raza Bulldog inglés.

\section{MATERIALES Y MÉTODOS}

Un canino Bulldog inglés, hembra, de 6 años, con historia clínica de muerte súbita y diagnóstico presuntivo de envenenamiento, se remitió al Departamento de Ciencias Patológicas de la Facultad de Ciencias Veterinarias de la Universidad Nacional de Asunción, para estudio post-mortem. Evaluándose macroscópicamente al corazón junto con la bolsa pericárdica. Debido a la presencia de sangre intrapericárdica, se procedió a la colecta y medición del volumen de sangre extravasada $(450 \mathrm{ml}$ ) (Figura 1), subsecuentemente se evaluó la existencia de una protuberancia única, irregular, localizada en la base del corazón y alrededor de la aorta ascendente (Figura 2). Se extrajeron de la cavidad torácica el corazón y la formación tumoral aórtica, obteniéndose posteriormente las medidas. Los fragmentos 
tisulares de la aurícula izquierda y la formación tumoral de la base del corazón, así como fragmentos del hígado, riñón y pulmón, se colocaron para su fijación en formol bufferado al 10\% por 48 horas. Todas las muestras obtenidas se remitieron al Laboratorio de Histopatología de la FCV-UNA para su procesamiento con la técnica histológica de rutina de inclusiones en parafina, teñidas con hematoxilina y eosina (HyE) y seccionadas con un grosor de $5 \mu \mathrm{m}$.

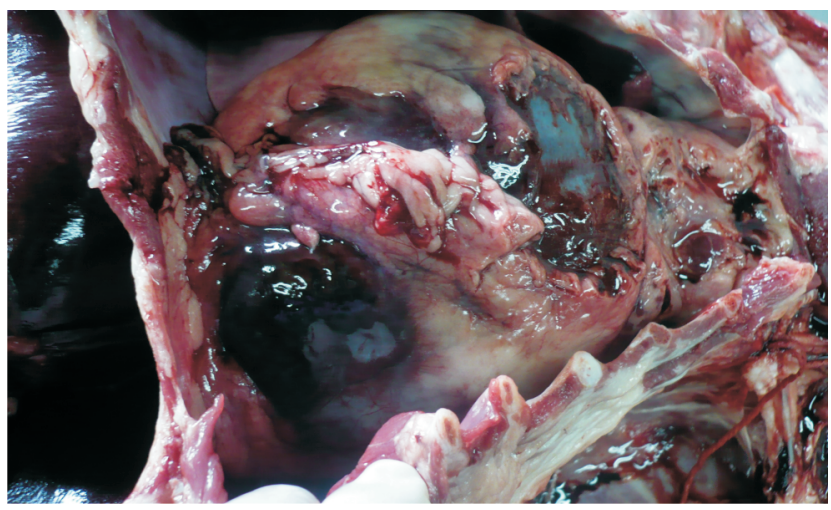

Figura 1. Hemopericardio agudo.

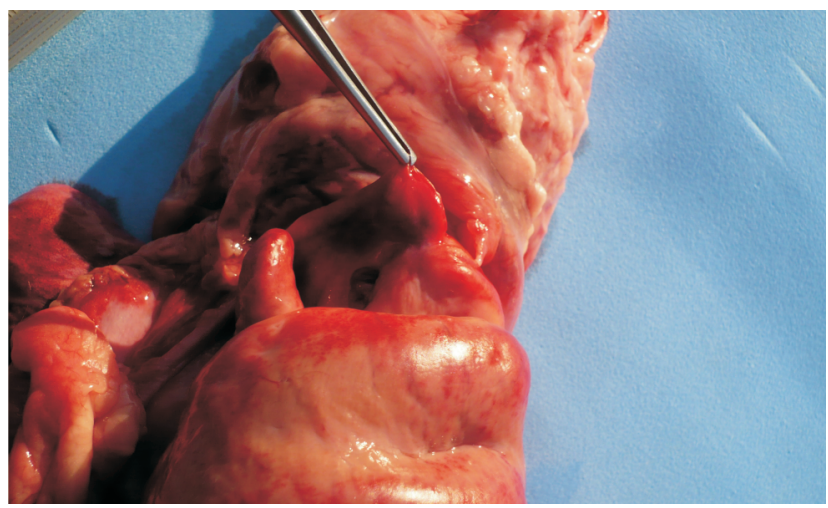

Figura 2. Superficie de corte de masa de la base del corazón y aorta ascendente.

\section{RESULTADOS Y DISCUSIÓN}

La evaluación post-mortem reveló mucosas conjuntivales cianóticas, fosas nasales con secreción sanguinolenta, tejido subcutáneo y muscular de la región cervical con hemorragias maculosas, además de abundante tejido adiposo en el tejido subcutáneo de todo el cuerpo, así como en pericardio y cavidad torácica, además del omento y mesenterio de la cavidad abdominal. En la cavidad torácica, la bolsa pericárdica se apreció redondeada, congestionada y hemorrágica, tensa y elástica, abarcando casi el $80 \%$ del espacio torácico. A la apertura, se colectaron aproximadamente $450 \mathrm{ml}$ de sangre, de color rojo oscuro y con coágulos, el corazón se encontró agrandado, con marcada dilatación de los vasos coronarios y engrosamiento de las paredes del miocardio del lado del ventrículo izquierdo con una medida de $1,3 \mathrm{~cm}$ y del lado derecho de $0,5 \mathrm{~cm}$, la pared auricular izquierda de color blanco-grisáceo con una medida de $0,3 \mathrm{~cm}$ y la pared auricular del lado derecho con $0,2 \mathrm{~cm}$. Asimismo, la luz del ventrículo del lado izquierdo presentó una medida de 10,4 cm y la del ventrículo del lado derecho $6,9 \mathrm{~cm}$. Una formación tumoral irregular con una medida de 4 x 2,5 cm, se encontró en la base del corazón periférica a la aorta, continuándose con la adventicia e infiltrando la pared de la aurícula izquierda con dos perforaciones irregulares en la base y cara lateral del tumor (Figura 3). Al corte, la neoplasia de color blanco-grisáceo y consistencia friable, presentó zonas hemorrágicas próximas al área de necrosis central (Figura 4). Los pulmones mostraban un color rojo oscuro. En la cavidad abdominal, el hígado se observó de color rojo oscuro, con restos de fibrina adheridos a la cápsula hepática. Los riñones se encontraron congestionados, con aparentes estrías radiales blanquecinas, y en pelvis.

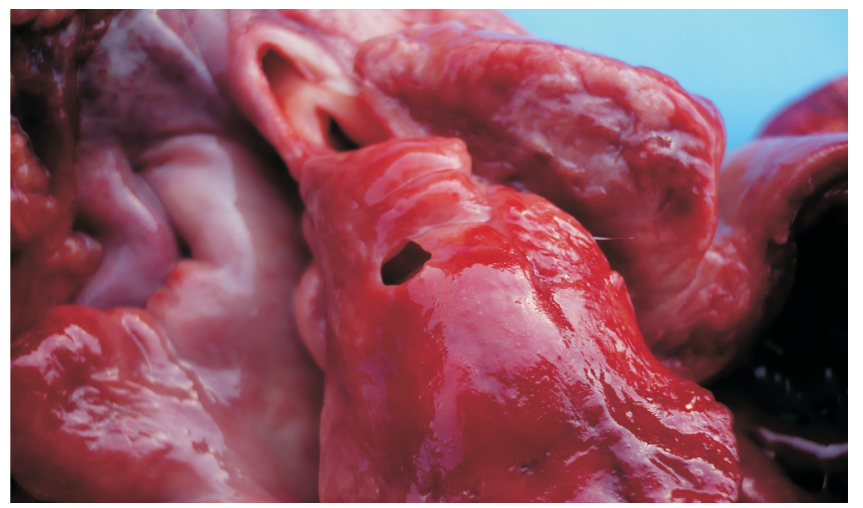

Figura 3. Formación tumoral con perforación central.

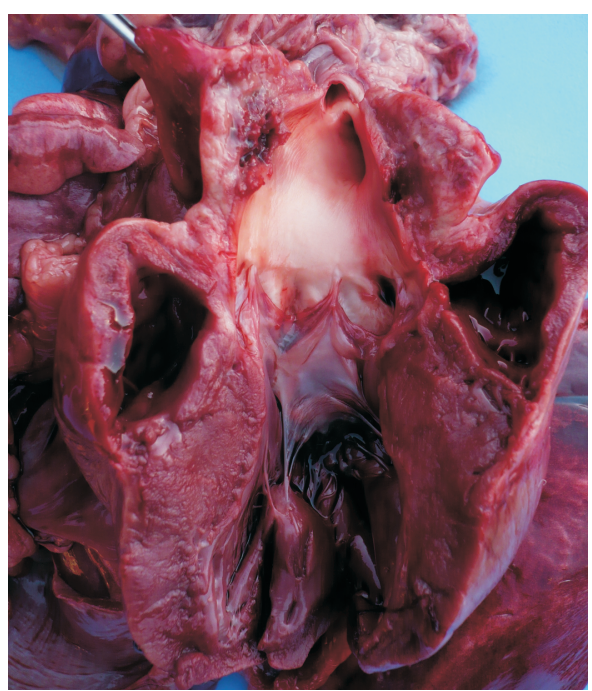

Figura 4. Superficie de corte de masa tumoral con hemorragia y necrosis central. 
De todas las especies de animales domésticos, son en los caninos los que con mayor frecuencia se diagnostica este tipo de tumor, le siguen los gatos y después los bovinos (11). Las razas Bulldog, así como los Bóxer y los Boston Terrier, son considerados como los más susceptibles a padecer con frecuencia este tipo de tumor $(3,8,11,12,13)$.

Algunos autores sugieren que el desarrollo de la patogenia, es debida a una hipoxia crónica a consecuencia de la estructura de las vías aéreas altas en caninos braquiocefálicos, que conlleva a una hipertrofia y finalmente a una transformación neoplásica de las células quimiorreceptoras (8). Así también, una predisposición al desarrollo de este tumor fue detectado y descrito en animales e inclusive humanos, que habitan en montañas altas donde la presión parcial de oxígeno es baja $(3,8)$.

La evaluación histopatológica de la masa tumoral reveló que la misma se encontraba dividida en lóbulos por tejido conjuntivo que se originaba de una delgada cápsula con múltiples invaginaciones y vasos sanguíneos hiperémicos. Los lóbulos estaban subdivididos por colágeno y fibras reticulares adelgazadas, principalmente en las porciones profundas, junto a células poliédricas, redondeadas y ovales con citoplasma granular (Figura 5).

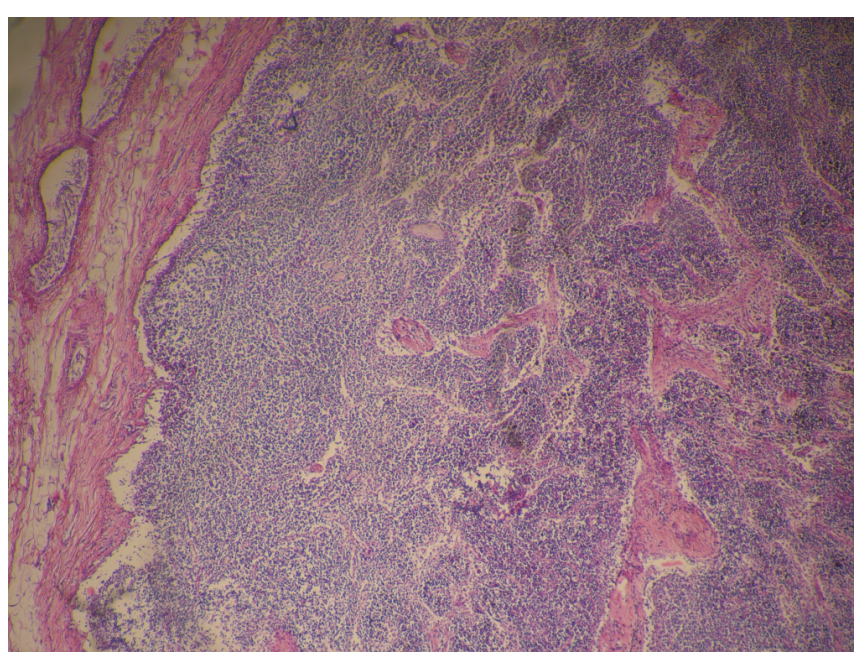

Figura 5. Células neoplásicas organizadas en lóbulos subdivididas por colágeno y fibras reticulares (H-E) 20x.

El miocardio auricular izquierdo, reveló infiltrado de células neoplásicas con intenso pleomorfismo celular y nuclear de características similares a las células evaluadas en la masa (Figura 6).

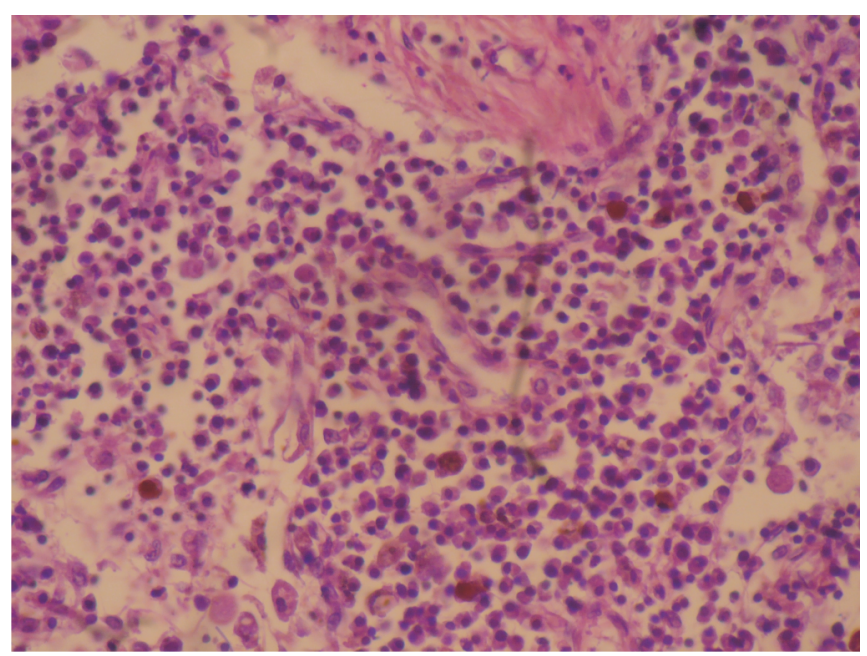

Figura 6. Infiltrado de células neoplásicas pleomórficas en miocardio auricular izquierdo (H-E) 100x.

Los quimiodectomas ocurren usualmente como tumores no malignos, crecen por expansión y en raras ocasiones infiltran tejidos adyacentes, realizando en ocasiones metástasis en pulmón, hígado, miocardio, cerebro y hueso (8). En este caso se describe la presencia de infiltrado de células tumorales en miocardio auricular izquierdo destacándose así el carácter histológico y comportamiento maligno biológico del tumor.

Casos de quimiodectomas en caninos fueron descritos en varios estudios siendo la infiltración auricular izquierda por células neoplásicas reportada sólo en uno de ellos $(3,7,8,9,10,11,12)$.

La presencia de hemopericardio e hidropericardio ha sido descrita en algunos estudios $(2,7,9)$. Sin embargo, en otros reportes mencionan ausencia de hemopericardio $(7,8,10,12)$.

Este tipo de neoplasia ha sido más frecuentemente diagnosticado en machos que en hembras, sin embargo, en el presente estudio el diagnóstico fue en una hembra $(3,7,8,9,10,11,12)$.

\section{CONCLUSIÓN}

Los hallazgos macroscópicos y microscópicos confirmaron que la muerte súbita del paciente, está asociada a un cuadro de insuficiencia cardiaca congestiva, así como a dos perforaciones de la arteria aorta, que dieron origen a un hemopericardio agudo, shock hipovolémico y colapso cardiopulmonar. 
Los quimiodectomas son neoplasias raras en caninos, con esporádicos reportes de casos en la literatura veterinaria. Generalmente son tumores no funcionales, que ocasionan falla cardiaca congestiva y hemopericardio, debido a la necrosis experimentada por las células neoplásicas.

\section{BIBLIOGRAFÍA}

1. Graciano T, Bettini C, Headley S, Monteiro E, Quitzan J. Paraganglioma de corpo aórtico e carotídeo. Arq. Cienc. Vet. Zool. UNIPAR. julio de 2009; 12(2): 149-156.

2. Headley S, Bracarense A, Nakagawa T, Moreno K, Mendes P, Faria dos Reis A. Aortic body tumors in dogs from Northern Parana. Cien. Rural. septiembre de 2009.; 39(6):1908-1912.

3. Genneser F. Histología. 3a ed. Madrid: Panamericana; 2012.

4. Blain G, Smith C, Henderson K, Dempsey J. Peripheral chemoreceptors determine the respiratory sensitivity of central chemoreceptors to C02.J Physiol. 2010; 588(13): 2455-2471.

5. Aresu L, Tursi M, Iussich S, Guarda F, Valenza F. Use of S-100 and chromogranin A antbodies as inmunohistochemical markers on detection of malignancy in aortic body tumors in dog. J.Vet. Med. Sci. 2006; 68 (11): 1229-1233.

6. González C, Rocher A, Zapata P. Quimiorreceptores arteriales: Mecanismos celulares y moleculares de las funciones adaptativas y homeostática del cuerpo carotídeo. Rev. Neurol. 2003; 36: 239-254.

7. Noszczyk A, Nowak M, Paslawska U, Atamaniuk W, Nicpon J. Cases with manifestation of chemodectoma diagnosed in dogs in Department of Internal Dieseases with Horses, Dogs an Cats clinic, Veterinary Medicine Faculty, University of Environmental and Life Sciences, Wroclaw, Poland. Acta Vet. Scand. 2010; 52(35): 1-7.

8. Nowak M, Noszczyk-Nowak A, Mróz K, Dziegel P. Infiltrative form of a tumour in cardiac base in a dog with dilated cardiomyopathy: clinical and morphological correlations. A case report. Bull Vet Inst Pulawy. 2008; 52:485-490.

9. Perrone EA, Xavier JG, Chamas P, Catão J. Chemodectoma in dogs: a case report. Braz. J. Vet Res. Anim. Sci. 1992; 29(2): 233237.

10. Eriksson M. Aortic body tumors in dogs. Sveriges lantbruksuniversitet. 2011;2:1-11.

11. Balaguer L, Romano J, Nieto J, Vida S, Alvarez C. Incidental finding of a chemodectoma in a dog: differential diagnosis. J. Vet. Diagn. Invest. 1990; 2: 339-341.

12.Meuten DJ. Tumors in Domestic Animals. Blackwell Publishing. 4a ed. USA: State Avenue, Ames, Iowa; 2002. 2121 p.

13. Atasever A, Cam Y. Aortic ody Tumor in a Dog. Turk J. Vet. Anim. Sci. 2003; 27: 1241-1245. 